\title{
Autonomes Fahren und Stadtstruktur
}

\author{
Dirk Heinrichs
}

\section{Inhaltsverzeichnis}

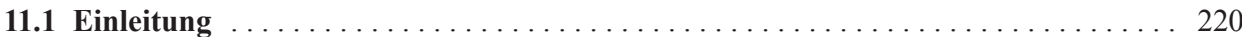

11.2 Autonomes Fahren als Bestandteil von Szenarien zur Stadt von morgen . . . . . . 221

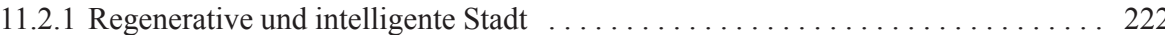

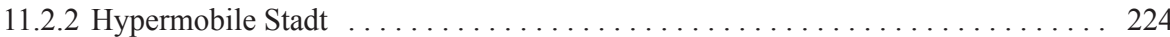

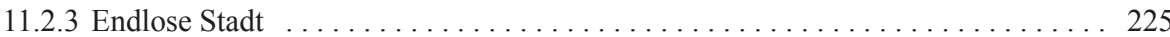

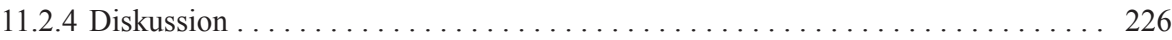

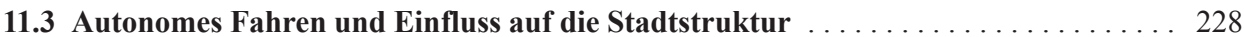

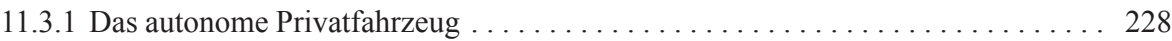

11.3.2 Das autonome Taxi als integrierter Teil des öffentlichen Verkehrs . . . . . . . . 232

11.4 Wesentliche Treiber für die Entwicklung eines Verkehrssystems

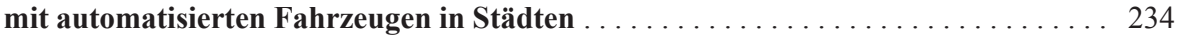

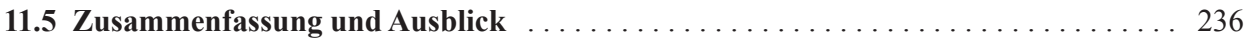

11.5.1 Aspekte in der Diskussion um automatisiertes Fahren aus Sicht

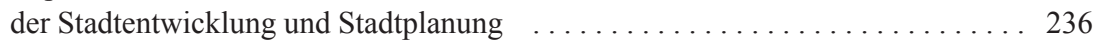

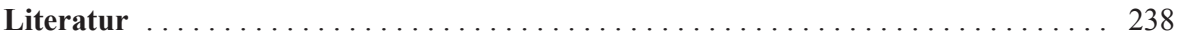

D. Heinrichs $(\bowtie)$

Deutsches Zentrum für Luft- und Raumfahrt (DLR), Institut für Verkehrsforschung, Deutschland Dirk.Heinrichs@dlr.de 


\subsection{Einleitung}

Mobilität, Verkehr und die physische Gestalt städtischer Räume sind eng miteinander verknüpft [1]. Die Stadtstruktur bildet eine wichtige Grundlage für Mobilitätsentscheidungen von Haushalten und Unternehmen und gibt in entscheidendem Maße vor, welche Formen von Verkehr ermöglicht oder aber auch ausgeschlossen werden. Kompakte Stadtstrukturen mit hoher Dichte und Nutzungsmischung bieten gute Voraussetzungen für kurze Wege, ein leistungsfähiges öffentliches Verkehrsangebot, fördern den Fuß- und Radverkehr und machen die Nutzung des Kraftfahrzeugs im Alltag oft unnötig. Ist die Stadtstruktur hingegen zersiedelt und weist sie eine geringe Dichte auf, werden Fuß- und Radverkehr erschwert, und der motorisierte Individualverkehr wird begünstigt. Die Verfügbarkeit und die Nutzung von bestimmten Verkehrsmitteln üben wiederum einen starken Einfluss auf städtische Strukturen und die notwendigen Infrastrukturen aus. So wurde die Wohn-Suburbanisierung der zweiten Hälfte des letzten Jahrhunderts in starkem Maß durch die Verfügbarkeit des Pkw und den Ausbau der Verkehrsinfrastruktur für den motorisierten Individualverkehr gefördert [2].

Erwartungen gehen davon aus, dass mit dem vollautomatisierten Fahren ein ganz neues Verkehrssystem entsteht, das nicht nur neue Möglichkeiten der Verkehrssteuerung mit sich bringt, sondern auch ganz neue Beförderungsangebote generiert, welche die Wahl und Nutzung von zur Verfügung stehenden Verkehrsmitteloptionen beeinflussen (s. Kap. 12). Die Vorstellung, dass beispielsweise die Zeit im Fahrzeug nicht mit Fahraufgaben verbracht werden muss, sondern andere Aktivitäten zulässt, kann eine vollständige Neubewertung des Faktors Zeit nach sich ziehen (z. B. [28]). Dies kann dazu führen, dass Nutzer für ihre täglichen Wege andere (weiter entfernte) Ziele in Betracht ziehen oder gar ihre Wohnstandortwahl verändern, da lange Pendelwege nicht mehr als nachteilig gesehen werden. Denkt man diese Zusammenhänge konsequent weiter, so wäre letztlich die Auflösung des Faktors Zeit als begrenzende Variable der Stadtentwicklung möglich. Wird also die Verfügbarkeit vollautomatisierter Fahrzeuge das Wechselverhältnis zwischen Mobilität und städtischen Strukturen völlig neu definieren? Zu dieser Frage existieren mit wenigen Ausnahmen [3] noch keine Vorstellungen. Die Visionen zur Integration des autonomen Fahrzeugs in das städtische Verkehrssystem beziehen sich derzeit im Wesentlichen noch auf die Entwicklung der Fahrzeugtechnologie selbst und die Auswirkungen auf den Verkehrsfluss.

Vor diesem Hintergrund ist es Ziel dieses Beitrags, die möglichen stadtstrukturellen Entwicklungen unter dem Einfluss eines Verkehrssystems mit autonomen Fahrzeugen auszuloten. Gleichermaßen soll abgeschätzt werden, in welcher Weise politische und ökonomische Rahmenbedingungen diese Entwicklungen beeinflussen können. Die folgenden Fragen stehen dabei im Vordergrund:

- Welche zukünftigen Möglichkeiten eines durch automatisiertes Fahren veränderten bzw. veränderbaren Verkehrssystems sind denkbar?

- Welche Auswirkungen auf Stadtstrukturen, insbesondere auf deren Dichte, Nutzungsmischung und Gestaltung, könnten zukünftig damit verbunden sein? 
- Welche Einflussfaktoren sind besonders bedeutsam für die Entwicklung eines Verkehrssystems mit automatisierten Fahrzeugen in Städten?

- Welche Aspekte sollten in die Diskussion um das automatisierte Fahren aus Sicht der Stadtentwicklung und Stadtplanung zwingend mit einfließen, aber auch: In welcher Weise sollte die Diskussion um die Entwicklung der Städte das Thema „Automatisierung des Verkehrs“" aufgreifen?

Als Basis für die Untersuchung dieser Fragen wertet der Beitrag verfügbare aktuelle Szenarien zur Stadt der Zukunft aus. Der folgende Abschn. 11.2 stellt die in der Literatur verfügbaren Szenarien bzw. Visionen zur „Stadt von morgen“ und deren Vorstellungen bezüglich einer Integration von Verkehrssystemen mit autonomen Fahrzeugen vor. Auf dieser Grundlage beschreibt er, welche unterschiedlichen zukünftigen Möglichkeiten eines durch automatisiertes Fahren veränderten bzw. veränderbaren Verkehrssystems denkbar sind, in welchen städtischen Strukturen sie sich entwickeln und welche wesentlichen Einflussfaktoren die Autoren für die Entwicklung zugrunde legen.

Aufbauend darauf führt Abschn. 11.3 eine vertiefte Analyse anhand von zwei vom Autor entwickelten idealisierten Szenarien des zukünftig denkbaren ,automatisierten Verkehrssystems“ durch. Kern dieser Szenarien ist a) das autonome Privatfahrzeug; b) das autonome Fahrzeug als integrierter Teil des öffentlichen Verkehrs. Auf der Grundlage einer kurzen Charakterisierung beider Szenarien wird analysiert, welche zukünftigen Auswirkungen auf Stadtstrukturen mit dem jeweiligen Szenario verbunden sein könnten.

Abschnitt 11.4 lotet aus, welche Einflussfaktoren besonders bedeutsam für die Entwicklung eines Verkehrssystems mit automatisierten Fahrzeugen in Städten sein könnten. Für die vorgestellten Szenarien werden die wesentlichen Treiber benannt.

Der abschließende Abschn. 11.5 fasst die wesentlichen Erkenntnisse zusammen. Er diskutiert, welche Aspekte in die Diskussion um das automatisierte Fahren aus Sicht der Stadtentwicklung und Stadtplanung zwingend mit einfließen sollten, aber auch, in welcher Weise der Diskurs um die Stadtentwicklung und -planung das autonome Fahren aufgreifen sollte.

\subsection{Autonomes Fahren als Bestandteil von Szenarien zur Stadt von morgen}

Da automatisiertes Fahren in Städten gegenwärtig noch nicht Realität ist und damit Wirkungen auf Stadtstrukturen derzeit noch nicht beobachtbar sind, bieten Szenarien eine Möglichkeit, denkbare zukünftige Entwicklungen und Zusammenhänge heranzuziehen. Szenarien beschreiben sowohl eine denkbare zukünftige Situation als auch die Entwicklung des Weges, der aus dem Heute in die Zukunft hineinführt [4, 5]. Sie stellen ein anerkanntes Instrument dar, mit dem sich Veränderungen, ihre Treiber sowie die Folgen in einem teilweise unbekannten, unsicheren und sich rasch ändernden Umfeld aufdecken und strukturieren lassen [6]. 
Für die nachfolgende Darstellung erfolgte eine systematische Auswertung verfügbarer Studien zur Stadt der Zukunft, in denen die Entwicklung der Mobilität bzw. des Verkehrs thematisiert wird. Aus der Gesamtheit der Dokumente wurden Kerndokumente und darin dargestellte Szenarien ausgewählt, die folgende Kriterien erfüllen: eine nachvollziehbare Darstellung eines Zielzustands und eines Entwicklungspfades, die Identifikation von Triebkräften und ihren Interdependenzen, die Behandlung des Themas Mobilität und Darstellung der Wechselwirkungen mit der Siedlungsstruktur. Diese Dokumente wurden im Anschluss zu Typen vergleichbarer Ausprägungen zusammengefasst und dahingehend ausgewertet, welche Formen und Bedeutung automatisierten Fahrens beschrieben werden bzw. welche Veränderungen diese Entwicklung maßgeblich prägen. Die Abgrenzung zueinander erfolgte entlang zweier Unsicherheitsachsen, die für die Auseinandersetzung mit dem automatisierten Fahren besonders relevant sind: die Verfügbarkeit und Integration intelligenter Kommunikationsinfrastruktur (gering bzw. hoch) sowie die Akzeptanz und Nutzung dieser Infrastruktur allgemein bzw. für die Mobilität durch die städtische Bevölkerung (gering bzw. hoch).

Die in den als Kerndokumenten eingestuften beschriebenen Szenarien lassen sich im Wesentlichen drei Typen zuordnen:

- regenerative und intelligente Stadt,

- hypermobile Stadt,

- endlose Stadt.

Nachfolgend werden die Szenarien näher erläutert.

\subsubsection{Regenerative und intelligente Stadt}

Eine Reihe von Zukunftsstudien heben die Entwicklung sogenannter regenerativer Städte als einen denkbaren Entwicklungspfad hervor [7, 8, 9, 10, 11, 12]. Diese Studien sehen für den Zeitraum 2030-2050 technologische Entwicklungen als Kern und Triebkraft städtischer Veränderung, welche den Ressourceneinsatz effizient und umweltverträglich gestalten. Im Mittelpunkt stehen der energetische Umbau von Gebäuden (Solarisierung, Plus-Energiehäuser) und die zunehmende Nutzung dezentral erzeugter Energien aus erneuerbaren Quellen, welche über sogenannte Microgrids bzw. Peer-to-peer-Energiesysteme verteilt und geteilt werden. Unterstützt werden sie durch intelligente Steuerungsmechanismen, die eine Verknüpfung mit anderen Bereichen städtischer Funktionen wie Mobilität ermöglichen. Die Bedeutung von Intelligenz und Information wird in den entsprechenden Studien hervorgehoben. Sie beschreiben den Wandel hin zu einem technologischen Regime, welches nicht mehr durch Technologien für einzelne Sektoren (Energie, Verkehr, Abfall etc.) charakterisiert wird, sondern ein hohes Maß an Integration zwischen den Sektoren gewährleistet.

Im Zuge dieser technologischen Entwicklung vollzieht sich ein Verhaltenswandel der städtischen Bevölkerung [7]. Es wird allgemein ein nachhaltiger Konsum als sehr bewuss- 
ter und verantwortlicher Umgang mit Ressourcen angenommen [9]. Erklärt wird dies durch den in Zukunft stärker werdenden Wunsch der konsumierenden Stadtbevölkerung nach Wohlbefinden und Lebensqualität, die sich anders definiert als über ökonomischen Wohlstand. Von der Gesellschaft wird eine energieoptimierte, nachhaltige und zukunftsfähige Mobilität getragen, weil die Mehrheit der Bevölkerung deren Vorteile nicht nur in den Medien präsentiert bekommt, sondern im eigenen Alltag erleben kann.

Der Schlüssel dazu liegt in den dicht bewohnten Metropolregionen, in denen eine vielfältig vernetzte und dennoch einfache und bezahlbare Mobilitätsversorgung gewährleistet ist. Städte werden in diesen Szenarien als Orte charakterisiert, die aufgrund ihrer Dichte eine effiziente Ressourcennutzung ermöglichen. Hinzu kommt die Annahme, dass mit der wachsenden Bedeutung von Städten als ökonomische und soziale Zentren die Entscheidungs- und Handlungspotenziale auf städtischer Ebene in Zukunft zunehmen. Eine Reihe der analysierten Studien verweist darauf, dass Städte wie London bereits heute demonstrieren, dass sich städtische Infrastrukturen umgestalten und dezentrale innovative Ansätze für Energie und Abfall umsetzen lassen, ohne dass nationale Politik erforderlich ist. Städte wandeln sich, getrieben durch Wettbewerb (untereinander) sowie durch Politik und Verwaltung, welche aktiv daran arbeiten, die Standortqualitäten zu verbessern.

Im Zuge des ressourceneffizienten Umbaus der Städte wandeln sich auch die Bedingungen für Mobilität in der regenerativen und intelligenten Stadt. Das Verkehrssystem erfährt eine zunehmende Durchdringung mit Informations- und Kommunikationstechnologie. Darin sehen die analysierten Studien die Grundlage für den Ausbau bedarfsorientierter Mobilitätsmanagementansätze und die Verknüpfung von Verkehrsangeboten zu einem künftigen flexiblen, multimodalen Verkehrssystem (s. Kap. 9). Der ÖPNV als Rückgrat der städtischen Mobilität wird weiter ausgebaut und laufend modernisiert, in integrierter Planung im Umweltverbund mit Fuß- und Fahrradwegen, deren Anteil am Straßenraum zunimmt. Ergänzend dazu stehen den Bürgerinnen und Bürgern individuell nutzbare Verkehrsmittel (Fahrräder, e-Bikes, Elektroautos, Elektrotransporter) zur Verfügung, und zwar zeitlich dann und örtlich dort, wann und wo der individuelle Bedarf besteht (mobility on demand). Diese „Sharing“-Angebote nach dem Prinzip „nutzen statt besitzen“ werden von verschiedenen Anbietern auf- und ausgebaut und helfen, die derzeit vom motorisierten Individualverkehr beanspruchte Fläche im öffentlichen Raum stark zu verringern. Ein persönlicher, mobiler und elektronischer Mobilitätsassistent ermöglicht es, alle für die tägliche Mobilität zur Verfügung stehenden Handlungsalternativen abzuwägen und situationsspezifisch optimale Varianten auszuwählen.

Die Weiterentwicklung elektronischer Assistenzsysteme für Pkw wird in den Studien im Zusammenhang mit der Entwicklung effizienterer neuer Antriebsformen thematisiert. Es wird davon ausgegangen, dass das eigene Fahrzeug auch in Zukunft seine Bedeutung behalten wird [11]. Verschiedene Studien erwarten die Präsenz von Fahrzeugen im Zeitraum 2030-2050, in denen fest eingebaute elektronische Assistenzsysteme für teilautonome Nutzungen zur Standardausrüstung gehören. Beispielsweise auf Autobahnen mit hohem Transitaufkommen oder Pendlerstrecken ermöglichen sie die Fahrt mit dem Autopiloten, womit eine Optimierung des Verkehrsflusses erreicht wird. Sichergestellt wird dies durch 
eine hohe Vernetzung und Kommunikation zwischen Fahrzeug und Verkehrsinfrastruktur. Diese Entwicklung wird flankiert durch neue Gesetzgebung bezüglich Zulassung, Haftungsrecht und Versicherungswesen sowie durch ein Akzeptanz schaffendes Konzept des Staates zu den Themen Datenmanagement und Standardisierung von Daten (Open Source, Schnittstellenkompatibilität, Datenschutz und Sicherheit).

Im Zuge der Veränderungen des Mobilitätsangebots beschreiben die untersuchten Studien auch einen Wandel der räumlichen Struktur der Städte. Die Verknüpfung von Verkehrsangeboten, so die Autoren, verstärkt die bereits heute zu beobachtende Herausbildung von sogenannten Mobilitätsknoten. Es wird davon ausgegangen, dass sich in Zukunft Stadtquartiere um diese Knoten herum in einer polyzentrischen Stadtstruktur organisieren. Der Flächenverbrauch für Stellflächen im Stadtraum hat sich deutlich reduziert. Die Gründe hierfür sind eine dynamische Verteilung der Fahrzeuge in Stadtquartieren sowie automatisierte „Parkregale“, die ein platzsparendes Abstellen von Fahrzeugen sicherstellen.

\subsubsection{Hypermobile Stadt}

Die hypermobile Stadt als ein denkbarer Entwicklungspfad wird insbesondere in einer Studie des Foresight Directorate des UK Office of Science and Technology thematisiert [7]. Beschrieben wird die Entwicklung einer Gesellschaft bis in das Jahr 2055, in der kontinuierliche Information, Konsum und Wettbewerb die Norm sind [7].

Auch dieses Szenario geht, wie im Fall der regenerativen Stadt, davon aus, dass durch technologische Entwicklung und Innovation derzeit noch existierende Barrieren zum individuellen Nutzen einer Mehrheit der Gesellschaft überwunden werden, aber mit einem weiterhin sehr hohen Bedarf an Ressourcen und den entsprechenden Umweltfolgen. Ein wesentliches Element und Treiber für die Entwicklung ist die Akzeptanz der Entwicklung elektronischer und digitaler Infrastruktur, wie beispielsweise die Nutzung von Kameras für den virtuellen Austausch oder von persönlichen Informationsassistenten. Ist diese Akzeptanz derzeit noch gering, so nimmt sie aufgrund ihrer Vorteile für Lebensstil und Handel in den kommenden Jahrzehnten deutlich zu. Menschen in der Stadt der Zukunft sind always on, ob zuhause oder am Arbeitsplatz. Dazu nutzen sie personalisierte, mit Verschlüsselungstechnologie ausgestattete Assistenten, mit deren Hilfe sie sich umfassend organisieren und ihren Alltag planen. Auch wenn in dem Szenario die Probleme und Vorbehalte bezüglich Datenschutz und Privatsphäre sehr deutlich thematisiert werden, so werden diese letztlich aufgrund des Wertes der elektronischen Assistenten für die Nutzer an die Seite gedrängt.

Der Staat und der Privatsektor haben auf dem Weg in das Jahr 2055 miteinander kooperiert, um die erforderlichen Technologien zu entwickeln. $\mathrm{Zu}$ den treibenden Interventionen gehören die starke Förderung von nutzerbezogener Informations- und Kommunikationstechnologie (persönliche Assistenzsysteme, die Vereinheitlichung von Kommunikationsstandards und GPS) sowie Technologieentwicklung (Verschlüsselungstechnologien, Sensoren, Ortung). Eine wichtige Voraussetzung auf dem Weg sind starke Bemühungen um die Datensicherheit auf europäischer Ebene. 
Die Mobilität im Zieljahr dieses Szenarios (2055) wird als ausgesprochen vernetzt beschrieben. Die Autoren zeichnen eine Entwicklung, in der die Mobilitätsnachfrage weiter ansteigt. Die starke Ausrichtung auf Informations- und Kommunikationstechnologie hat in diesem Szenario auch die Automatisierung der Mobilität und des Verkehrs vorangetrieben, nicht zuletzt um die Verkehrsflüsse zu optimieren und Staus zu mindern. In den Städten ersetzen integrierte Massentaxi-Systeme weitgehend den gewöhnlichen öffentlichen Verkehr. Diese übernehmen das effiziente Abholen und Verteilen von Fahrgästen in Zustiegszonen. Die dafür eingesetzten lokalen Beförderungsfahrzeuge operieren in zugewiesenen Stadtgebieten und werden von Nutzern mithilfe ihres persönlichen Assistenten angefordert. Das Netzwerk kalkuliert die effizienteste Route, auch für das Abholen und Absetzen von mehreren Passagieren, und berechnet den Fahrpreis. Dieses auch als „Schwarm“ bezeichnete Netzwerk kann große Datenmengen über die Verkehrslage und Nachfragepositionen verarbeiten. Die Fahrzeuge können ihre Route anpassen. Passagiere können jedes Fahrzeug nutzen, anstatt auf eine bestimmte Linie warten zu müssen.

Autonome Fahrzeuge verkehren auf Autobahnen für Langstrecken auf einer eigens für sie reservierten guided lane, einige auch für Anwendungen über Nacht. Menschen kaufen größere Autos und fahren weitere Strecken. Diese Fahrzeuge sind mit einer on-board driverless unit ausgestattet, welche mit automatisierten Systemen entlang der Autobahnen sowie wesentlicher Pendlerrouten kommunizieren. In dieser Form entstehen Züge automatisch kontrollierter Fahrzeuge, welche mit hoher Geschwindigkeit eng zusammen fahren.

Die Entwicklung städtischer Strukturen wird in diesem Szenario differenziert beschrieben. Einerseits entstehen stark verdichtete Innenstädte, andererseits setzt sich das Wachstum suburbaner Gebiete geringer Dichte fort. Während vor allem jüngere Menschen die urbanen Zentren als Lebensort bevorzugen, entscheidet sich eine wachsende Zahl einkommensstarker Haushalte für einen Umzug an die Ränder der Städte oder in ländliche Gebiete. Trotz steigender Entfernung zu den in der Innenstadt gelegenen Arbeitsplätzen können sie weiter am intensiven Arbeitsleben teilnehmen, entweder mithilfe von Telepräsenz unter Nutzung noch leistungsfähigerer Kommunikationsinstrumente oder durch die bequeme Nutzung des automatisierten Fahrzeugs. Gleichzeitig verschafft sich diese Bevölkerungsgruppe durch das suburbane Wohnen das Gefühl und die Möglichkeit, sich von dem zunehmend anstrengenden und fordernden Arbeitsleben in der hypermobilen Welt zu erholen.

\subsubsection{Endlose Stadt}

Während die Szenarien der regenerativen und der hypermobilen Stadt die technologische Entwicklung als einen Motor für Veränderungen von städtischen Lebensweisen, Mobilität und städtischen Strukturen in den Vordergrund stellen, thematisieren andere Szenarien ein konträres Bild [12].

Die hier zugrunde liegenden Annahmen sind, dass sich technologische Innovationen nicht in dem umfassenden Maße durchsetzen, insbesondere aufgrund der hohen Kosten für 
erforderliche Infrastrukturen. Technologische Entwicklung findet zwar statt, ist aber vornehmlich auf Effizienzgewinne einzelner Bereiche (Verbrennungsmotoren, Solarenergie) beschränkt. Die Steuerungsmöglichkeiten des Staates werden als begrenzt gesehen. Auch ein Verhaltenswandel, wie in den vorherigen Entwicklungen skizziert, wird nicht sichtbar.

Hinsichtlich Mobilität und Raumstruktur sehen die Autoren dieses Szenarios ein autodominiertes Modell weiterhin deutlich ausgeprägt. Im öffentlichen Verkehr werden sich in vielen Städten aufgrund staatlich begrenzter Kapazitäten zur Weiterentwicklung des öffentlichen Verkehrssystems sogenannte informelle Paratransit-Dienste weiterentwickeln. Der Vernetzungsgrad mit vorhandenen Angeboten bleibt gering. Die Möglichkeit eines autonomen Verkehrssystems wird nicht thematisiert. Räumlich sind Städte durch eine niedrige Dichte und fragmentierte Siedlungsstrukturen gekennzeichnet. Diesbezüglich schreiben die Autoren einen derzeit global zu beobachtenden Trend fort [13].

\subsubsection{Diskussion}

Die hier dargestellte Auswahl und Analyse zeigt, dass Szenarien die Möglichkeit eines durch automatisiertes Fahren veränderten bzw. veränderbaren Verkehrssystems teilweise thematisieren. Automatisierte Lösungen werden insbesondere in Szenarien mit hoher Durchdringung und Verknüpfung innovativer Kommunikations- und Ortungstechnologie formuliert (regenerative und hypermobile Stadt). Automatisiertes Fahren im städtischen Kontext wird dabei als Beitrag im öffentlichen Verkehr erwartet. Die Szenarien beschreiben beispielsweise den Einsatz von stapel- und programmierbaren Kleinstfahrzeugen oder ein integriertes Massentaxi-System. Das automatisierte Privatfahrzeug findet im Zuge des Langstreckenverkehrs auf Autobahnen Erwähnung (s. Tab. 11.1).

Mit Blick auf die Auswirkungen durch automatisiertes Fahren auf die Stadtstruktur beschreiben die unterschiedlichen Szenarien zunächst den Zusammenhang mit einem allgemeinen Wandel der Rahmenbedingungen. Das Szenario der regenerativen Stadt zeigt sich in einer zunehmenden Dichte von Bevölkerung und Funktionen in Städten. Andere Szenarien (hypermobile und endlose Stadt) gehen von einer Fortführung derzeit global zu beobachtender Suburbanisierungstendenzen als Folge individueller Präferenzen einkommensstarker Haushalte oder aufgrund von Abdrängungsprozessen einkommensschwächerer Haushalte aus. Als sichtbare Veränderung der Stadtstruktur durch ein Verkehrssystem mit Elementen des automatisierten Fahrens wird in verschiedenen Szenarien die Entstehung sogenannter Mobilitätshubs oder -knoten beschrieben. Im Szenario der regenerativen und intelligenten Stadt wird die Vorstellung der Vernetzung konsequent auf den Stadtraum übertragen. Multimodale Verkehrsknoten ermöglichen eine physische Vernetzung und einen einfachen Umstieg zwischen verschiedenen Modi wie beispielsweise vom (Elektro-) Auto auf öffentliche Verkehrsmittel. Das Szenario geht noch einen Schritt weiter, indem es über die Bündelung unterschiedlicher Mobilitätsangebote auch von einer Änderung sonstiger Nutzungen ausgeht. Es beschreibt Stadtquartiere, die sich rund um Mobilitätsknoten und Versorgungsdienstleistungszentren organisieren und bei denen automatisierte Fahr- 
Tab. 11.1 Die Szenarien im Überblick

\begin{tabular}{|c|c|c|c|}
\hline Szenario & $\begin{array}{l}\text { Ausprägung autonomen } \\
\text { Fahrens }\end{array}$ & Stadtstruktur & Treiber \\
\hline $\begin{array}{l}\text { Regenerative } \\
\text { Stadt }\end{array}$ & $\begin{array}{l}\text { - flexibles, multimoda- } \\
\text { les und vernetztes } \\
\text { öffentliches Verkehrs- } \\
\text { system als Rückgrat } \\
\text { der städtischen } \\
\text { Mobilität } \\
\text { - (teil)-autonome Pkw } \\
\text { (Autopilot) auf Auto- } \\
\text { bahnen }\end{array}$ & $\begin{array}{l}\text { Herausbildung } \\
\text { von intermodalen } \\
\text { Mobilitätsknoten } \\
\text { - Reduktion des } \\
\text { Flächenverbrauchs für } \\
\text { Stellflächen im } \\
\text { Stadtraum durch neue } \\
\text { Parksysteme }\end{array}$ & $\begin{array}{l}\text { - technologische Ent- } \\
\text { wicklung (im Energie- } \\
\text { system) } \\
\text { - bewusster und ver- } \\
\text { antwortlicher Umgang } \\
\text { mit Ressourcen } \\
\text { - Gesetzgebung und } \\
\text { Akzeptanzförderung } \\
\text { durch den Staat }\end{array}$ \\
\hline $\begin{array}{l}\text { Hypermobile } \\
\text { Stadt }\end{array}$ & $\begin{array}{l}\text { hoch integrierte } \\
\text { (autonome) Massen- } \\
\text { taxi-Systeme } \\
\text { - autonome Pkw auf } \\
\text { Autobahnen mit } \\
\text { hohem Transitauf- } \\
\text { kommen oder Pendler- } \\
\text { strecken auf reservier- } \\
\text { ten guided lanes }\end{array}$ & $\begin{array}{l}\text { - stark verdichtete } \\
\text { Innenstädte } \\
\text { - Wachstum suburbaner } \\
\text { Gebiete geringer } \\
\text { Dichte }\end{array}$ & $\begin{array}{l}\text { - zunehmende Akzep- } \\
\text { tanz von Informations- } \\
\text { und Kommunikations- } \\
\text { technologien aufgrund } \\
\text { ihrer Vorteile für Le- } \\
\text { bensstil und Handel } \\
\text { - Kooperation von Staat } \\
\text { und Privatsektor, } \\
\text { um erforderliche IKT- } \\
\text { Technologien } \\
\text { zu entwickeln }\end{array}$ \\
\hline Endlose Stadt & $\begin{array}{l}\text { - autodominiertes } \\
\text { Modell vorherrschend } \\
\text { geringe Integration } \\
\text { des öffentlichen Ver- } \\
\text { kehrs (hoher Anteil } \\
\text { informeller Para- } \\
\text { transit-Angebote } \\
\text { - keine nennenswerten } \\
\text { Entwicklungen hin } \\
\text { zu automatisiertem } \\
\text { Fahren }\end{array}$ & $\begin{array}{l}\text { - Wachstum suburbaner } \\
\text { Gebiete } \\
\text { - generelle Abnahme } \\
\text { der Siedlungsdichte }\end{array}$ & $\begin{array}{l}\text { - fehlende Steuerungs- } \\
\text { fähigkeit des Staates } \\
\text { - technologische Ent- } \\
\text { wicklung beschränkt } \\
\text { auf Effizienzgewinne } \\
\text { einzelner Bereiche }\end{array}$ \\
\hline
\end{tabular}

Eigene Darstellung, basierend auf [7-12]

zeuge als Teil der öffentlichen Fahrzeugflotte einbezogen sind. Auch das Parken und seine Verknüpfung mit dem Raum werden in nahezu allen Szenarien in sehr unterschiedlicher Weise thematisiert. Das Szenario der regenerativen Stadt beschreibt den Rückgang des Flächenbedarfs im Zusammenhang mit dem sinkenden Anteil privater Pkw und einem dezentralen Parkraummanagement. In der intelligenten Stadt der Zukunft hat sich eine Verknüpfung der Nutzung des eigenen Pkws mit dem öffentlichen Verkehr durchgesetzt, und es entstehen neue P+R-Flächen an Mobilitätsknoten.

Insbesondere die Beschreibungen zur intelligenten und regenerativen Stadt sind dabei geprägt durch die Grundüberzeugung, dass die Technologie derzeit existierende bzw. ab- 
sehbare Probleme (Ressourcenknappheit, Umweltwandel) überwinden wird. Die zentrale Bedeutung technologischer Entwicklung wird auch im Szenario der endlosen Stadt bestätigt, allerdings aus der umgekehrten Perspektive. Dort wird das Fehlen von Innovation als Erklärung für eine Reihe negativer Entwicklungen interpretiert. Dieses Szenario steht in gewissem Sinne für eine denkbare Entwicklung in Städten des globalen Südens, in denen die Steuerungskapazitäten des Staates als vergleichsweise gering eingestuft werden.

Eine kritische Auseinandersetzung mit dem Thema Daten erfolgt lediglich im Szenario der hypermobilen Stadt. Probleme des Datenschutzes und der Sicherheit gelten dort im Zielzeitraum um 2050 als gesellschaftlich akzeptiert, da die individuellen Vorteile der Informations- und Kommunikationslösungen für die Teilhabe in sozialen Netzwerken und im Arbeitsleben die Nachteile aus Sicht der Bevölkerung überwiegen.

\subsection{Autonomes Fahren und Einfluss auf die Stadtstruktur}

Die im vorherigen Abschnitt dargestellten Szenarien entwickeln eine Vorstellung davon, in welcher Form das automatisierte Fahren in der Stadt der Zukunft als ein Bestandteil des Verkehrssystems denkbar ist. Im Folgenden wird ein näherer Blick auf die möglichen Veränderungen der Stadtstruktur gelegt, die sich in dem Zusammenhang ergeben: Wie ändern sich Verteilung von Nutzungen, Dichte und Gestaltung städtischer Räume unter dem Einfluss autonomen Fahrens?

Die genannten Szenarien zeigen, dass voneinander grundsätzlich unterscheidbare Optionen denkbar sind. Zum einen beschreiben sie die Entwicklung eines autonomen Privatfahrzeugs, welches je nach Szenario „bordautonom“ durch einen Autopiloten gesteuert wird oder durch Fahrzeug-Infrastruktur-Kommunikation in den Verkehrsfluss eingebunden ist. Zum anderen sehen die Szenarien das autonome Fahren als integrierten Teil des öffentlichen Verkehrsangebots. Es ist davon auszugehen, dass die Wirkungen auf die Stadtstruktur abhängig von der Ausprägung des autonomen Verkehrssystems sehr unterschiedlich sein dürften. Daher werden nachfolgend beide Ausprägungen einzeln behandelt.

\subsubsection{Das autonome Privatfahrzeug}

Diese Ausprägung beschreibt im Kern die Übertragung von Fahraufgaben auf Automaten im unimodalen Individualverkehr. Sie greift Aspekte der in Kap. 2 dargestellten Anwendungsfälle Autobahnpilot, Vollautomat mit Verfügbarkeitsfahrer und Valet-Parken auf.

Dieser Fall geht davon aus, dass die zukünftige Nutzung mit der heutigen Pkw-Nutzung weitgehend übereinstimmt. Abgesehen von den veränderten Eigenschaften der eingesetzten Technologie werden keine Veränderungen angenommen. Nach wie vor ist der Pkw in individuellem Besitz. Es werden keine Annahmen zu Änderungen des Modalwahl- und Zielwahlverhaltens getroffen. Allerdings gibt es Änderungen gegenüber der derzeitigen Nutzung des Fahrzeugs. Zum einen ermöglicht autonomes Fahren andere Aktivitäten wäh- 
rend der Fahrt: Vormalige Fahrer können nun - beispielsweise - auf ihrem Laptop arbeiten, essen, ein Buch lesen, einen Film anschauen oder Freunde anrufen [14]. Zum anderen verändern sich die Zugangssituation und die Abgangssituation. Während der Pkw heutzutage entweder direkt vom Wohnort zum Zielort gefahren wird bzw. der Nutzer vor Fahrtantritt das Fahrzeug aufsucht und nach Fahrtende die Strecke vom Parkplatz/-ort zum Ziel zurücklegt, wird unter dem Einfluss des autonomen Fahrens dieser Zu- und Abgangsweg durch einen Vor- bzw. Nachlauf des Fahrzeugs ersetzt. Der Fahrroboter manövriert das Fahrzeug von der ursprünglichen Parkposition zum Ort des Halters/Nutzers und nach Erreichen des Zielorts zu einer zugewiesenen Parkposition.

Die denkbaren Wirkungen bzw. Veränderungen durch den Einsatz eines autonomen Fahrzeugs auf die Stadtstruktur umfassen zum einen den benötigten Parkraum für Fahrzeuge am Wohnort sowie am Zielort. Zum anderen wird eine Veränderung der Attraktivität von Standorten als Wohnstandort von Haushalten erwartet, einen Attraktivitätswandel wird es aber auch bei Standorten geben, die Ziele für alltägliche Aktivitäten wie Einkaufen oder Freizeit sind. Hinzu kommt, dass sich mit dem Einsatz des autonomen Fahrzeugs der Flächenbedarf für den fließenden Verkehr verändert. Auf diese drei Aspekte wird nachfolgend näher eingegangen.

\subsubsection{Veränderung des benötigten Parkraums}

Die zu erwartenden Veränderungen des Parkraumbedarfs für Fahrzeuge am Wohnort sind insgesamt als gering einzuschätzen, unterscheiden sich allerdings abhängig von den Eigenschaften der Siedlungsstruktur. In Einfamilienhaus-Wohnsiedlungen, in denen sich der Stellplatz auf dem Wohngrundstück befindet, ist keine Veränderung zu erwarten. Hier wird der verfügbare Parkplatz lediglich durch ein anderes (autonomes) Fahrzeug belegt. In Gebieten hoher Dichte, wie beispielsweise in innerstädtischen Bereichen, ist von der Entwicklung bzw. Entstehung von wohnquartiersbezogenen Parkzonen oder Sammelgaragen auszugehen. Denn nur so kann sichergestellt werden, dass das autonom parkende Fahrzeug auch einen Platz im definierten Einzugsbereich des Wohnorts findet.

Weiterreichende Wirkungen auf den Parkraum durch den Einsatz autonomer Fahrzeuge sind am Zielort eines Weges denkbar, der nicht der Wohnort des Nutzers ist. Dies betrifft beispielsweise Einkaufswege, Wege zum Zweck der Erholung oder auch Arbeitswege. Autonome Fahrzeuge wären in der Lage, die Passagiere am Zielort abzusetzen und sich dann auf einem zugewiesenen Parkplatz oder auch in einer Sammelgarage eigenständig zu parken. Auch hier ist davon auszugehen, dass die entsprechenden Parkraumkapazitäten bereitgestellt werden, die dem Fahrzeug das Auffinden eines Parkplatzes sicher und verlässlich ermöglichen. Diese Anwendung autonomen Fahrens, bei der das Fahrzeug den Nutzer am Zielort absetzt und sich anschließend autonom parkt, dürfte einerseits mit einer höheren Akzeptanz bei Nutzern einhergehen, bestimmte Ziele mit dem Pkw aufzusuchen. Andererseits dürften sich erhebliche Effekte bezüglich der Bereitstellung und Bewirtschaftung von Parkraum ergeben. Insbesondere in Gebieten hoher Nutzungsdichte ist davon auszugehen, dass eine Bündelung des Parkraumangebots in Form von Sammelgaragen erfolgt. 
Die Möglichkeit zur Einsparung von Fläche wird als ein wesentliches Argument für den Einsatz automatischer Parksysteme genannt, wobei die günstige Flächenausnutzung in erster Linie durch den Ersatz von Rampen und Fahrgassen durch Fördervorrichtungen sowie die Verringerung der Geschosshöhen erreicht wird, aber auch durch das dichte Einparken $[14,15]$. Bei Einsatz des Parkroboters gehen die Entwickler von bis zu 60 Prozent mehr Parkplätzen auf gleicher Fläche aus [16].

Die effizientere Nutzung von Flächen für das Parken ist insbesondere aus Kostengesichtspunkten attraktiv [16, 17, 18]. Die für ein Bauvorhaben zu errichtenden Stellplätze haben einen hohen Flächenbedarf und stellen, vor allem wenn die Stellplätze nicht ebenerdig errichtet werden, einen nicht unerheblichen Anteil an den Kosten der Gesamtinvestition dar. Es gibt bereits erste Projekte, die das Parken in Parkhäusern mit autonomen Fahrzeugen testen [19, 20]. Der Fahrer übergibt das Fahrzeug am Eingang des Parkhauses. Die Parkfunktion wird mithilfe einer Smartphone-App aktiviert. Über WLAN erhält das Auto die Routendaten vom Zentralrechner im Parkhaus zum nächsten freien Parkplatz und fährt autonom dorthin. Als ein Vorbild können hierfür bereits heute existierende automatische Parksysteme dienen, bei denen abgesehen von der Ein- und Ausfahrt in einer Übergabekabine alle notwendigen Fahrzeugbewegungen automatisch über Fördervorrichtungen bzw. Verschiebeeinrichtungen realisiert werden, oder mit eigens entwickelten Parkrobotern, wie sie beispielsweise am Flughafen Düsseldorf bereits im Einsatz sind [16].

Wie erwähnt, dürfte sich die Restrukturierung und mögliche Konzentration von Parkflächen nicht flächendeckend durchsetzen, sondern vorrangig auf Gebiete beschränken, die als Ziel besonders attraktiv sind und deren Kosten für die Erstellung erforderlicher Parkflächen und damit die Anreize für platzsparende Lösungen besonders hoch sind (Baulandpreise, Flächenknappheit und damit Bedarf an mehrgeschossigen Lösungen). Hierzu zählen hoch verdichtete innerstädtische Dienstleistungs- und Einkaufszentren oder auch neue Gewerbegebiete mit hoher Zahl an Beschäftigten. Hinzu kommen Mobilitätsknoten - oft auch mit dem englischen Begriff $H u b$ bezeichnet - wie Flughäfen oder Bahnhöfe, an denen neben den vorherigen Kriterien auch sichere Lösungen für das längere Einstellen von Fahrzeugen gefordert sind. Inwieweit sich im Zuge der Automatisierung die Kosten für das Parken insgesamt verändern dürften, ist nicht abzuschätzen. Einerseits kann das Absetzen von Passagieren am Zielort und anschließendes Parken an anderer Stelle im Stadtgebiet (mit etwas niedrigerem Nutzungsdruck und damit günstiger) Kosten sparen [21, 22]). Andererseits entstehen mit dem Umbau des Parkraumangebots für autonomes Fahren wiederum Kosten.

\subsubsection{Veränderung der Attraktivität von (Wohn-)Standorten}

Mit der Verfügbarkeit autonomer Fahrzeuge sind auch Effekte auf die Art der Flächennutzung denkbar. Einige Studien weisen auf den Zusammenhang einer steigenden Attraktivität von Wohngebieten am Stadtrand hin [2, 22, 23]. Nach dieser Argumentation kann die Verfügbarkeit eines autonomen Fahrzeugs dazu führen, dass Haushalte im Grünen gelegene, kostengünstigere, aber weiter vom Zentrum der Stadt entfernt gelegene Standorte wählen, da das autonome Fahrzeug die Standortnachteile (größere Entfernung) kompen- 
siert. Eine Folge wäre die Entstehung von neuen Siedlungsgebieten vergleichsweise geringer Dichte und geringer Nutzungsmischung analog zur Suburbanisierung in der zweiten Hälfte des vergangenen Jahrhunderts. Dort haben Motorisierung, Infrastruktur und die planerisch-politischen Leitbilder der Nutzungstrennung und der aufgelockerten Stadt sowie die Entscheidung von Haushalten für die Ansiedlung im Grünen eine bis heute prägende Siedlungsstruktur geschaffen [24].

Generell ist bekannt, dass die Wohnstandortwahl von Berufstätigen durch Faktoren wie Wohn- und Wohnumfeldqualität weitaus stärker beeinflusst wird als durch den Wunsch nach Nähe zum Arbeitsplatz [25]. Erkennbar wird dies durch eine vergleichsweise hohe Bedeutung des Arbeitspendelns. Etwa 60 Prozent aller sozialversicherungspflichtig Beschäftigten in Deutschland, das sind rund 17 Millionen Personen, arbeiten nicht in der Gemeinde, in der sie wohnen. Um zum Arbeitsort zu gelangen, benötigen Beschäftigte durchschnittlich ca. eine halbe Stunde pro Weg. Dabei dominiert die Pkw-Nutzung, indem etwa 66 Prozent der Wege mit dem Auto zurückgelegt werden [26], in den USA sind es sogar 86 Prozent [27]. Auf Basis von Datensätzen zur Erwerbstätigkeit und Beschäftigung sowie zu regionalen Berufsverflechtungen haben Guth et al. [25] herausgefunden, dass in den Agglomerationsräumen in Deutschland der Anteil der gemeindeübergreifenden Berufspendelwege und die zurückgelegten Distanzen in den letzten Jahrzehnten angestiegen sind.

Autonomes Fahren könnte diesen Trend und die Bereitschaft zur Inkaufnahme längerer Pendel-Arbeitswege weiter fördern. Zum einen wird angenommen, dass sich mit dem autonomen Fahren eine Steigerung des Fahrkomforts verbindet (z. B. [14]). Die Fahrzeit muss nicht mehr für die aufmerksame und verantwortungsvolle Fahrzeugführung aufgewendet werden, sondern kann anderen Aktivitäten dienen. Mobilität wird nicht notwendigerweise als Zwang oder Zeitverlust empfunden. Des Weiteren lassen sich Fahrzeiten verkürzen. Im Zusammenhang mit dem autonomen Fahren bestehen hohe Erwartungen an eine allgemein effizientere Abwicklung des fließenden und ruhenden Verkehrs [2, 22, 28]. Autonome Fahrzeuge können ihre Fahrweise miteinander harmonisieren, beispielsweise bei Beschleunigungs- und Bremsvorgängen, und damit Wegezeiten reduzieren. Ebenso werden nahezu keine Verzögerungen mehr an Kreuzungen vorausgesagt [14]. Auch beim Parksuchverkehr werden deutliche Reduzierungen des Zeitbedarfs erwartet, bedingt durch das Absetzen von Passagieren. Die Fahrt mit dem autonomen Fahrzeug kann insgesamt prognostizierbarer und zeitlich verlässlicher geplant werden. Dies ergibt sich aus nahezu konstanten Geschwindigkeiten und einer verlässlichen und vorhersagbare Route vom Ausgangspunkt zum Ziel.

Verbesserter Fahrkomfort, verringerte Wegezeiten und höhere Verlässlichkeit von Reisezeiten sind relevante Faktoren für die Abwägung von Haushalten, weiter entfernte Arbeitsplätze oder sonstige Ziele wie Versorgungs- und Bildungseinrichtungen zugunsten anderer Kriterien eines Wohnstandorts wie die Bezahlbarkeit des Wohnraums oder die landschaftliche Attraktivität des Umfeldes. Besonders für Berufspendler dürfte sich mit der Verfügbarkeit eines autonomen Fahrzeugs und der prognostizierten Effekte die Entscheidungsgrundlage verändern. Insbesondere Wegezeiten stellen eine hohe Zusatzbelastung für 
Pendler dar [29]. Dies wird dort noch verstärkt, wo die Zeitdauer und damit das Erreichen des Ziels schwer kalkulierbar werden. Psychologische Studien [30] zeigen, dass Zeitverluste, die der Verkehrsteilnehmer nicht selbst kontrollieren kann - beispielsweise durch Stau - in besonders hohem Maße für Stress verantwortlich sind.

\subsubsection{Flächenbedarf für den fließenden Verkehr}

Die genannten Vorteile des autonomen Fahrens lassen auch erwarten, dass Kapazitäten von Verkehrswegen frei werden. Aufeinander abgestimmte Beschleunigungs- und Bremsvorgänge und die dichtere Fahrzeugfolge (das sogenannte Platooning) ermöglichen eine Reduzierung des Straßenraums zur Abwicklung des Fahrvorgangs [30]. Dadurch wird eine deutlich höhere Fahrzeugdichte bezogen auf die Fläche des Straßenraums erwartet [30, 31], wobei unterschiedliche Aussagen zum Umfang dieser Kapazitätssteigerung existieren. So geht Fernandez von bis zu 500 Prozent aus [31]. Brownell schätzt gut 250 Prozent für Autobahnen und etwa 180 Prozent für innerstädtische Straßen [32]. Dies bedeutet, dass die für den fließenden Verkehr erforderliche Fläche reduziert werden könnte, beispielsweise durch die Reduktion von Fahrspuren. Auch die Breite von Fahrstreifen könnte, aufgrund des veränderten Fahrzeugverhaltens autonom fahrender Fahrzeuge, gegenüber gegenwärtigen Maßen reduziert werden (s. Kap. 16). Aufgrund der Reduzierung des Flächenbedarfs durch den fließenden Verkehr könnten andere Nutzungen gefördert werden, wie beispielsweise Fahrstreifen für Fahrräder oder Fußwege. Allerdings weisen verschiedene Autoren darauf hin, dass diese Effekte erst bei vollständiger Automatisierung zum Tragen kommen [22].

Die Verdichtung des fließenden Verkehrs könnte aber auch in anderer Weise Auswirkungen auf Verkehrsteilnehmer wie Fußgänger und Radfahrer nach sich ziehen. So dürfte die Trennungswirkung zunehmen und das Queren von Fahrspuren unter Bedingungen eines dicht fließenden Verkehrs erschwert werden. Um die Vorteile des autonomen Fahrens für den Verkehrsfluss zu gewährleisten und gleichzeitig die „Durchlässigkeit“ für den Fuß-und Radverkehr sicherzustellen, wäre die Anlage von kreuzungsfreien Querungen wie Überoder Unterführungen eine notwendige Konsequenz.

\subsubsection{Das autonome Taxi als integrierter Teil des öffentlichen Verkehrs}

Eine zweite Entwicklung, die weitaus prominenter in den dargestellten Szenarien thematisiert wird, ist die Entstehung eines neuen Modells städtischer Mobilität in Form autonomer Taxiflotten. Sie greift Aspekte des in Kap. 2 beschriebenen Anwendungsfalls ,Vehicle-onDemand" auf. In einem solchen System operieren kostengünstige autonome Taxis nicht auf festen Routen und nach starren Fahrplänen, sondern bedarfsorientiert und flexibel. Sie sind im permanenten Fahrbetrieb und verkehren in einem stadtweiten, dichten Netz von Stationen. Die Funktionsweise ähnelt dem Anruf-Sammeltaxi. Das Stadtgebiet ist in Zellen aufgeteilt. Zu jeder Zelle gehört eine oder eine Reihe von zentralen Aus- und Zustiegsstationen, sogenannte central transit points. Denkbar ist, dass Taxis mit dem schienen- 
gebundenen öffentlichen Verkehrsangebot (ÖV) verknüpft sind bzw. kombiniert werden können. Die Taxis übernehmen die Zubringer- bzw. Feinverteilungsfunktionen zu den Stationen des schienengebundenen ÖV und nehmen dort Fahrgäste auf, während der leistungsfähigere und möglicherweise schnellere ÖV die langen Streckenabschnitte übernimmt. Der Einsatz eines automatisierten Taxi-Netzwerks ist als Konzept bereits beschrieben und modelliert [32, 33, 34]). Es könnte zu einem grundlegenden Wandel des öffentlichen Nahverkehrs führen und das Problem der letzten Meile von Hochgeschwindigkeitsbahnen lösen [23]. Es beinhaltet den Wegfall klassischer Bus- und Tram-Haltestationen. Der Betrieb der Taxis könnte öffentlich oder privat erfolgen.

\subsubsection{Verkehrs- und Parkflächen des öffentlichen Raums}

Die Auswirkungen eines öffentlichen Verkehrssystems mit autonomen Fahrzeugen auf Stadtstrukturen dürften sehr weitreichend sein. Der Einsatz autonomer Fahrzeuge könnte die Anzahl von Parkplätzen in Stadtzentren deutlich reduzieren. Denn nach Absetzen von Passagieren müsste sich das Fahrzeug nicht zu einer entfernteren Station zum Parken navigieren, sondern würde einfach zum nächsten Passagier weiterfahren. Damit sind mehr Fahrzeuge im kontinuierlichen Betrieb, und der Stellplatzbedarf sinkt. Erforderlich ist allerdings die Einrichtung dezentraler Depots für Reinigung, Wartung, Tanken/Laden oder Reparatur der eingesetzten Fahrzeuge.

Der permanent verfügbare Einsatz einer solchen Taxiflotte dürfte car sharing und möglicherweise auch dynamisches ride sharing erhöhen, da es spontanes und minutenbzw. entfernungsgenaues Mieten eines Fahrzeugs für die Tür-zu-Tür-Fahrt ermöglicht [14]. Es könnte als eine konsequente Weiterentwicklung bereits heute existierender flexibler Carsharing-Geschäftsmodelle gesehen werden, welche diese Eigenschaften bereits besitzen (Car2go, City Car Club, DriveNow, Zipcar). Vor diesem Hintergrund könnte davon ausgegangen werden, dass ein solches System Fahrzeugbesitz und -nutzung spürbar verändern wird (s. Kap. 9). Auch eine Steigerung des Besetzungsgrades der Pkw-Nutzung ist denkbar [22].

Eine Folge könnte ein Rückgang des Pkw-Besitzes in Haushalten sein, die im Einzugsgebiet eines solchen Mobility-on-demand-Angebots leben. So könnten sich US-Amerikaner derzeit vorstellen, ihren Zweitwagen bei Verfügbarkeit eines solchen Systems mit Direktabholung vor der Haustür abzuschaffen [28]. Durch die Veränderung der Pkw-Besitzraten würde sich der Stellplatzbedarf verändern. Dies könnte so weit gehen, dass der ruhende Verkehr weitgehend zugunsten multifunktionaler Wegflächen entfällt. Diese Flächen könnten breiter als bisherige sein und unterteilt in einen befahrbaren Raum für Fahrräder und möglicherweise elektrisch unterstützte Mikrofahrzeuge mit Geschwindigkeiten bis etwa $30 \mathrm{~km} / \mathrm{h}$ und in eine Fahrbahn für schwerere und schnellere Fahrzeuge.

Eine weitere Veränderung der Gestalt städtischen Raums ist die Einrichtung von Ausund Zustiegsstationen, welche die Ausprägung von Mobilitätsknoten weiter fördert, insbesondere solche mit Umstiegsmöglichkeiten zum sonstigen ÖV. Über die Umgestaltung dieser Räume zum Halten, zur Aufnahme von Passagieren oder zum Kurzparken hinaus 
sind Nutzungsänderungen zu erwarten, wie beispielsweise die Zunahme und Konzentration von Dienstleistungs- und Einkaufsgelegenheiten an den Knoten.

Durch die weitreichende Umgestaltung des öffentlichen Verkehrssystems sind auch Auswirkungen auf die komplementären ÖV-Infrastrukturen und -nutzungen denkbar. Ein Umstieg von Nutzern vom eigenen Pkw auf ein autonomes Taxiangebot, welches mit einem leistungsfähigen schienengebundenen Liniennetz verknüpft ist, würde das Passagieraufkommen auf diesen Strecken stärken und sehr wahrscheinlich Kapazitätsanpassungen nach sich ziehen müssen.

\subsection{Wesentliche Treiber für die Entwicklung eines Verkehrs- systems mit automatisierten Fahrzeugen in Städten}

In den vorangegangenen Abschn. 11.2 und 11.3 wurden vorhandene Szenarien analysiert und anhand unterschiedlicher Ausprägungen die Wirkungen eines durch autonomes Fahren beeinflussten Verkehrssystems auf die Stadtstruktur diskutiert. Anknüpfend daran hinterfragt dieser Abschnitt, welche Einflussfaktoren besonders bedeutsam für die Entwicklung eines Verkehrssystems mit automatisierten Fahrzeugen in Städten sein könnten.

Die Diskussion der Szenarien zeigt zunächst die herausragende Bedeutung technologischer Innovationen, in deren Zusammenhang autonomes Fahren eine zunehmende Rolle im Verkehrssystem erlangt. Fortschritte und neue Entwicklungen im Bereich der Informations- und Kommunikationstechnologie, der elektronischen und digitalen Infrastruktur, des Datenmanagements und der künstlichen Intelligenz sind wesentliche Treiber dieses Trends, wobei die Automatisierung der Verkehrsmittel als Teil einer „umfassenderen“ Automatisierung von Prozessen in Städten gedeutet werden kann. Beispiele hierfür sind die Automatisierung des Parkens oder des Energie- und Gebäudemanagements.

Damit einhergehend zeigt sich, welche hohen Erwartungen an die Steuerungskapazitäten des Staates mit der Entwicklung eines Verkehrssystems mit autonomen Fahrzeugen verbunden sind. Der Staat kooperiert mit dem Privatsektor, um die erforderlichen Technologien zu entwickeln. Er flankiert dies durch eine neue Gesetzgebung bezüglich Zulassung, Haftungsrecht und Versicherungswesen sowie durch ein Akzeptanz schaffendes Konzept zu den Themen Datenmanagement und Standardisierung von Daten.

Hinsichtlich der Akzeptanz autonomen Fahrens durch die Bevölkerung wird deutlich, dass eine Reihe denkbarer Faktoren positiven Einfluss nehmen könnten. Zum einen verbindet sich mit dem autonomen Fahren der gesellschaftliche Nutzen eines effizienteren und umweltschonenderen Verkehrssystems. Zum anderen ist es durchaus vorstellbar, dass die Akzeptanz von privaten Nutzern und wirtschaftlichen Akteuren aufgrund der individuellen Vorteile für Lebensstil oder Handel in den kommenden Jahrzehnten steigen wird, insbesondere unter Annahme einer sichtbaren Technologie- und Akzeptanzförderung durch den Staat.

Ein weiterer Faktor, der die Automatisierung positiv beeinflussen dürfte, ist die Perspektive einer kosteneffizienteren Nutzung und Aufwertung städtischen Raums. Ein Bei- 
spiel ist das Parken, für das eine Automatisierung deutliche Kostenersparnisse für die Erstellung von Parkflächen und aufgrund von Reduzierung des Flächenbedarfs Potenziale für Umwandlung in andere Nutzungen verspricht. Aber auch Flächen am Stadtrand oder im Umland werden im Zuge einer denkbaren Neubewertung von Standortwahlkriterien und steigender Attraktivität als Wohnstandort möglicherweise an Wert gewinnen.

Allerdings darf nicht darüber hinweggesehen werden, dass den genannten Faktoren große Unsicherheiten gegenüberstehen. Zunächst ist, angesichts grundsätzlicher rechtlicher und ethischer Fragen (s. Kap. 25 und Kap. 4), überhaupt nicht absehbar, in welcher Geschwindigkeit und in welcher Ausprägung sich städtische Verkehrssysteme mit autonomen Fahrzeugen entwickeln werden.

Auch die Folgen für den Verkehr und damit die Stadtstruktur sind damit derzeit noch nicht verlässlich prognostizierbar. Mit der Integration autonomen Fahrens als Teil eines kollektiven, öffentlichen Verkehrssystems dürften sich Carsharing und der Rückgang des Pkw-Besitzes stärker entwickeln. Eine verstärkte Akzeptanz und Nutzung des „eigenen“ autonomen Pkw dürfte die Perspektiven für den motorisierten Individualverkehr stärken. Insbesondere in letzterem Fall stellt sich die Frage nach möglichen „Rebound-Effekten“. Durch Zeiteinsparungen und geringere Nutzungskosten kann es zu einem höheren Konsum von Mobilität und zur Zunahme der Fahrleistung kommen [22]. Mit einer Zunahme autonomer Fahrzeuge wäre unter diesen Umständen eine Kompensation von Kapazitätsgewinnen die Folge.

Allerdings ist auch die Frage danach, wie sich die Höhe der zusätzlichen Kosten für die Nutzung autonomer Fahrzeuge entwickeln dürfte, derzeit noch nicht beantwortet. Die in diesem Kapitel analysierten Szenarien gehen auf diesen Punkt nicht plausibel ein, sondern argumentieren lediglich mit der Annahme, dass die Mobilität auch in der Zukunft bezahlbar sein wird. Kalkulationen zusätzlicher Kosten für die Ausrüstung von Pkw gehen jedoch davon aus, dass sich sowohl die Anschaffungs- als auch die Unterhaltungskosten zunächst deutlich erhöhen dürften [22, 28]). Hinzu kämen Folgekosten für Kommunen für die Anpassung von Verkehrsinfrastrukturen sowie für die Erschließung neuer Siedlungsgebiete in dem Fall, dass die Attraktivität von suburbanen Standorten in der Tat zunehmen würde.

Aus all diesen Aspekten folgt eine hohe Planungsunsicherheit für kommunale und regionale Akteure der Politik, der Verwaltungen, Verkehrsbetreiber sowie der Immobilienwirtschaft. Insbesondere die Anpassung von Infrastrukturen des Verkehrs und der Siedlungsentwicklung erfordert eine langfristige Herangehensweise einschließlich der entsprechenden Regulation und Finanzierung. Veränderungen der Raumstrukturen im Zuge autonomen Fahrens sind erst dann zu erwarten, wenn die meisten Fahrzeuge auf der Straße automatisiert sind. Solange dies nicht der Fall ist, dürften Verkehrsdichten nicht signifikant steigen, die Planbarkeit von Trips sich nicht verbessern, der Parkbedarf sich nicht wesentlich reduzieren und können Straßenquerschnitte nicht verringert werden. Damit fehlen zumindest den Akteuren kommunaler Verkehrs- und Stadtplanung derzeit noch wichtige Entscheidungs- und Handlungsgrundlagen nicht zuletzt auch vor dem Hintergrund fehlender Klarheit, in welcher Form sich autonomes Fahren mittel- bis langfristig durchsetzen wird. 


\subsection{Zusammenfassung und Ausblick}

Das Kernanliegen dieses Beitrags ist es, mögliche stadtstrukturelle Entwicklungen unter dem Einfluss eines Verkehrssystems mit autonomen Fahrzeugen auszuloten und abzuschätzen, in welcher Weise politische und ökonomische Rahmenbedingungen diese Entwicklungen beeinflussen können. Auf Basis verfügbarer Szenarien bzw. Visionen zur „Stadt von morgen“ und deren Vorstellungen bezüglich einer Integration von Verkehrssystemen mit autonomen Fahrzeugen zeigt er auf, dass unterschiedliche Entwicklungen denkbar sind: zum einen das autonome Privatfahrzeug, welches je nach Szenario „bordautonom“ durch einen Autopiloten gesteuert wird oder durch Fahrzeug-Infrastruktur-Kommunikation in den Verkehrsfluss eingebunden ist, oder zum anderen das autonome Fahren als integrierter Teil des öffentlichen Verkehrsangebots.

Abhängig von diesen unterschiedlichen Ausprägungen haben autonome Verkehrsmittel das Potenzial, das Verkehrssystem in ganz unterschiedlicher Weise zu verändern. Ihre Eigenschaften und Einsatzmöglichkeiten werden auch Auswirkungen auf Entscheidungen zur Entwicklung von Flächennutzungen und Raumstrukturen haben. Einflussbereiche umfassen beispielsweise den Bedarf und die Organisation des Parkens sowie die Attraktivität von Räumen als Wohnstandort, Einkaufs- oder Arbeitsziel. Darüber hinaus haben autonome Fahrzeuge das Potenzial, derzeit für Verkehrs- und Parkflächen genutzte Flächen in andere Nutzungen (sei es für andere verkehrliche Nutzungen wie Rad- oder Fußverkehr oder aber bauliche Nutzungen) zu überführen. Welche Veränderungen sich konkret vollziehen könnten, hängt dabei stark davon ab, in welche Richtung sich autonomes Fahren entwickeln wird.

\subsubsection{Aspekte in der Diskussion um automatisiertes Fahren aus Sicht der Stadtentwicklung und Stadtplanung}

Die Diskussion macht deutlich, dass eine Reihe möglicher Wirkungen autonomer Fahrzeuge auf Raumstrukturen existieren, die als Kriterien für die Entscheidung des Besitzes und der Nutzung autonomer Fahrzeuge relevant sind. Die Möglichkeit von Haushalten, ihre „suburbanen“ Präferenzen leichter zu realisieren, ist ein Beispiel hierfür. Entscheidungszusammenhänge zwischen längerfristigen (Standortwahl) und alltäglichen Mobilitätsentscheidungen (Wahl von Zielen und Verkehrsmitteln) sollten in die Diskussion um das autonome Fahren eingehen. Hinzu kommt, dass diesen individuellen Kriterien relevante Aspekte gegenüberstehen, die sich auf den gesellschaftlichen Nutzen einer Automatisierung des Verkehrssystems beziehen. Dies betrifft beispielsweise die Folgekosten für die Erschließung neuer Siedlungsgebiete am Stadtrand als Folge geänderter Attraktivität solcher Gebiete. Inwieweit sich diese Zusammenhänge mit der Einführung autonomen Fahrens tatsächlich realisieren und wie die Folgen zu bewerten sind, sollte verstanden und in der Diskussion um das automatisierte Fahren zwingend werden. 
Ein zweiter Aspekt betrifft die Erkenntnis, dass im Zuge der Veränderung des Verkehrssystems neben möglichen Änderungen von Flächennutzungen eine weitreichende Transformation hin zu neuen Infrastrukturen und umgestalteten Verkehrsflächen denkbar ist. Diese müssen die Entwicklungen der Fahrmuster und Verhalten von automatisierten Fahrzeugen und nicht automatisierten Fahrzeugen, aber auch andere Modi berücksichtigen. All das ist eine langfristige Aufgabe. Denn Veränderungen der Raumstrukturen im Zuge autonomen Fahrens sind erst dann zu erwarten, wenn die meisten Fahrzeuge auf der Straße automatisiert sind. Hier sind viele Fragen noch offen. So besteht ein Bedarf zur Analyse, wie autonomes Fahren die langfristigen Pläne zur Gestaltung von Parkflächen, Fahrradspuren, Kreuzungen, Bürgersteigen, Querschnittsgestaltungen von Straßen etc. beeinflussen könnte. Wesentlich für die Diskussion ist die Frage: Wie kann eine sukzessive Transformation hin zu einem Verkehrssystem mit autonomen Fahrzeugen gestaltet werden, und was sind überhaupt plausible Einführungsszenarien? Dabei wird nicht nur zu klären sein, wie vorhandene Infrastrukturen graduell „umgebaut“ werden können, sondern wie sich in diesem Zuge die derzeitige Bevorrechtigung und Rollen unterschiedlicher Modi und Verkehrsmittel verändern. Wie beschrieben, ist es vorstellbar, dass mit der Zunahme autonomer Fahrzeuge und der Einrichtung von speziellen Fahrspuren eine Entwicklung hin zu einer stärkeren Funktionstrennung zwischen den verschiedenen Modi einhergeht. Auch die Durchlässigkeit des Straßenraums für andere Verkehrsteilnehmer im Zuge eines dicht fließenden Verkehrs mit autonomen Fahrzeugen dürfte bei dieser Entwicklung verändert werden. Dies wirft die Frage auf, in welcher Form und wie gleichberechtigt die Nutzungsmischung unterschiedlicher Verkehrsmodi unter dem Einfluss autonomen Fahrens zukünftig organisiert werden sollte.

Spätestens hier wird deutlich: Nicht nur Aspekte der Stadtentwicklung sind relevant für die Diskussion um das autonome Fahren. Die Automatisierung des Verkehrssystems ist von großer Relevanz für Stadtplanung und -entwicklung und die hierfür entwickelten Leitbilder und Ziele. Inwieweit und unter welchen Voraussetzungen kann ein Verkehrssystem mit autonomen Fahrzeugen zur Realisierung derzeit gültiger Modelle wie die dichte und kompakte Stadt beitragen? Oder verknüpft sich damit die Aussicht auf die Rückkehr zur autogerechten Stadt? In welcher Beziehung stehen städtebauliche Elemente zur Strukturierung des autonomen Verkehrs zu den Anforderungen einer allenthalben propagierten Fußgänger-, Rad-, und Bahnstadt? Bedarf es unter dem Einfluss des automatisierten Fahrens der Formulierung grundlegend anderer bzw. neuer Leitbilder zur Entwicklung von Städten? Die Diskussion um Antworten auf diese Fragen kann beginnen. 


\section{Literatur}

1. Cervero, R., Kockelman, K. (1997): Travel demand and the 3Ds: Density, diversity, and design. In: Transportation Research Part D: Transport and Environment, Volume 2, Issue 3, September 1997, Pages 199-219. DOI: 10.1016/S1361-9209(97)00009-6

2. Apel, D. (2003): Der Einfluss der Verkehrsmittel auf Städtebau und Stadtstruktur. In: Bracher, T.; Haag, M. ; Holzapfel, H. ; Kiepe, F. ; Lehmbrock, M. ; Reutter, U. (Hrsg.): HKV - Handbuch der kommunalen Verkehrsplanung

3. RAND Corporation (2014): Autonomous Vehicle Technology. A Guide for Policymakers. URL: http://www.rand.org/pubs/research_reports/RR443-1.html; Zugriff am 2. Juli 2014

4. Wilson, I. H. (1978): Scenarios. In: Fowles J. und Fowles, R. B. (Hrsg.): Handbook of Futures Research. Greenwood Pub Group Inc, Westport und London, S. 225-247

5. von Reibnitz, U. (1992): Szenario-Technik. Instrumente für die unternehmerische und persönliche Erfolgsplanung. Gabler Verlag, Wiesbaden

6. Steinmüller, K. H. (1997): Grundlagen und Methoden der Zukunftsforschung - Szenarien, Delphi, Technikvorausschau. WerkstattBericht 21. Sekretariat für Zukunftsforschung. Gelsenkirchen. URL: http://steinmuller.de/media/pdf/WB\%2021\%20Grundlagen.pdf; Zugriff am 14. April 2014

7. Foresight Directorate (2006): Intelligent infrastructure futures. The Scenarios - Towards 2055 URL: http://www.bis.gov.uk/assets/foresight/docs/intelligent-infrastructure-systems/thescenarios-2055.pdf; Zugriff am 12. April 2014

8. Fraunhofer Gesellschaft zur Förderung der Angewandten Forschung e.V. (2012): Visionen zur Morgenstadt. Leitgedanken für Forschung und Entwicklung von Systeminnovationen für nachhaltige und lebenswerte Städte der Zukunft. URL: http://www.morgenstadt.de/de/_jcr_content/ stage/linklistPar/download/file.res/Fraunhofer_Visionen\%20zur\%20Morgenstadt_050212.pdf; Zugriff am 14. April 2014

9. Stadt Wien (Magistrat 18 der Stadtentwicklung und Stadtplanung) (2012): smart city Wien - towards a sustainable development of the city (= Blue Globe ReportSmartCities \#1/2012) URL: http://www.smartcities.at/assets/Projektberichte/Endbericht-Langfassung/BGR01-2012K11NE2F00030-Wien-v1.0.pdf; Zugriff am 12. April 2014

10. Bundesministerium für Bildung und Forschung (n.D.): Morgenstadt - eine Antwort auf den Klimawandel. URL: http://www.bmbf.de/pubRD/morgenstadt(1).pdf; Zugriff am 2.Mai 2014

11. Promotorengruppe Mobilität der Promotorengruppe Mobilität Wirtschaft - Wissenschaft (2013): Abschlussbericht der Promotorengruppe Mobilität. URL: http://www.forschungsunion.de/pdf/ mobilitaet_bericht_2013.pdf; Zugriff am 4. April 2014

12. Forum for the Future (2010): Megacities on the move. Your guide to the future of sustainable urban mobility in 2040. URL: http://www.forumforthefuture.org/sites/default/files/images/Forum/ Projects/Megacities/megacities_full_report.pdf; Zugriff am 12. April 2014

13. Angel, S., Parent, J., Civco, D. L., Blei, A. M. (2011): Making Room for a Planet of Cities. Lincoln Institute of Land Policy. Cambridge, MA

14. Fagnant, D. J., Kockelman, K. M. (2013): Preparing a Nation for Autonomous Vehicles: Opportunities, barriers and Policy Recommendations. Eno Foundation. URL: http://www.enotrans.org/wp-content/uploads/wpsc/downloadables/AV-paper.pdf; Zugriff am 12. April 2014

15. Irmscher, I. (n.D.): Benutzerfreundliche automatische Parksysteme - Besondere Anforderungen - Planung - Einsatz. URL: www.givt.de/index.php/de/component/jdownloads/finish/4/22; Zugriff am 14. April 2014

16. Kowalewski, S. (2014): Überlassen Sie das Parken Ray. Deutschlandradio Kultur. URL: http://www.deutschlandradiokultur.de/technologie-ueberlassen-sie-das-parken-ray.2165. de.html?dram:article_id=290092; Zugriff am 2. Mai 2014 
17. Mitchell, W.J., Boronni-Bird, E., Burns, L.D. (2010): Reinventing the Automobile. Personal Urban Mobility for the $21^{\text {st }}$ Century. The MIT Press. Cambridge, MA

18. LEG Stadtentwicklung GMBH (2008): Mobilitätshandbuch Zukunftsstandort Phoenix West. Dortmund. URL: http://www.mobilitaetsmanagement.nrw.de/cms1/download/mobilitaetshandbuch phoenix.pdf; Zugriff am 5. Mai 2014

19. Continental AG (2014): Der Fahrer entscheidet, das Auto übernimmt. URL: http://www.contionline.com/www/automotive_de_de/themes/passenger_cars/automated_driving

20. Audi (2012): Automatisch ins Parkhaus. URL: http://blog.audi.de/2012/09/21/automatisch-insparkhaus/; Zugriff am 2. Mai 2014

21. Litman, T. (2014): Ready or waiting. Traffic Technology International. January. 37-42. URL: http://www.traffictechnologytoday.com; Zugriff am 2. Mai 2014

22. Litman, T. (2014): Autonomous Vehicle Implementation Prediction. Implications for Transport Planning. URL: http://www.vtpi.org/avip.pdf; Zugriff am 14. Juni 2014

23. Le Vine, S., Polack, J. (2014): Automated Cars: A smooth ride ahead? ITC Occasional Paper-Number Five, February 2014. URL: http://www.theitc.org.uk/docs/114.pdf; Zugriff am 14. April 2014

24. Angerer, F., Habdler, G. (2008): Integration der Verkehrs- in die Stadtplanung. In: : Steierwald, G.; Künne, H. D. \& Vogt, W. (Hrsg.): Stadtverkehrsplanung. Grundlagen, Methoden, Ziele. 2. Auflage, Springer-Verlag. Berlin, Heidelberg. S. 18-28

25. Guth, D., Siedentop, S., Holz-Rau, C. (2012): Erzwungenes oder exzessives Pendeln? Zum Einfluss der Siedlungsstruktur auf den Berufspendelverkehr. In: Raumordnung und Raumforschung (2012) 70, S. 485-499

26. Destatis (Statistisches Bundesamt) (2014): Berufspendler: Infrastruktur wichtiger als Benzinpreis. URL: https://www.destatis.de/DE/Publikationen/STATmagazin/Arbeitsmarkt/2014_05/ 2014 05Pendler.html\#Link3; Zugriff am 12. April 2014

27. McKenzie, B. (2014): Modes Less Traveled - Bicycling and Walking to Work in the United States: 2008-2012. American Community Survey Reports. URL: http://www.census.gov/hhes/ commuting/; Zugriff am 5. April 2014

28. Silberg, G., Wallace, R., Matuszak, G. (2012): Self-Driving Cars: The Next Revolution, KPMG and the Centre for Automotive Research; URL: www.kpmg.com/Ca/en/IssuesAndInsights/ ArticlesPublications/Documents/self-driving-cars-next-revolution.pdf; Zugriff am 28. April 2014

29. Häfner, S., Rapp, H., Kächele, H. (2012): Psychosoziale Belastungen von Bahnpendlern und was soll man tun? In: Psychotherapeut 2012 (57): S. 343-351

30. Lewis, D. (2004): Commuters suffer extreme stress, in BBC, URL: http://news.bbc.co.uk/2/hi/ uk_news/4052861.stm; Zugriff am 4. Mai 2014

31. Fernandes, P. (2012): Platooning With IVC-Enabled Autonomous Vehicles: Strategies to Mitigate Communication Delays, Improve Safety and Traffic Flow. URL: http://home.isr.uc.pt/ pedro/ T-ITS-11-02-0065.pdf; Zugriff am 2. Mai 2014

32. Brownell, C. K. (2013): Shared Autonomous Taxi Networks: An Analysis of Transportation Demand in NJ and a 21st Century Solution for Congestion. URL: http://orfe.princeton. edu/ alaink/Theses/2013/Brownell,\%20Chris\%20Final\%20Thesis.pdf; Zugriff am 2. Mai 2014

33. Brownell, C. K., Kornhauser, A. (2013): Autonomous Taxi Networks: a fleet size and Cost Comparison between two emerging transportation models and the conventional automobile in the state of New Jersey. URL: http://orfe.princeton.edu/ alaink/TRB'14/TRB'14_BrownellPaper_0728v2. pdf; Zugriff am 14. April 2014

34. Gorton, M. (2008): Using Information Technology to Achieve a Breakthrough in Transportation in New York City. The Open Planning Project. August 2008. URL: http://www.streetsblog.org/ wp-content/pdf/SmartParaTransit.pdf; Zugriff am 2. Mai 2014 